\title{
I principali aspetti biologici del volo spaziale, con particolare riguardo al microclima della cabina (*)
}

\author{
A. SCANO $(* ;)$
}

Ricevuto il 19 Febbraio 1963

\begin{abstract}
Ressivento. - Dopo un cenno ai principali aspetti biologici del volo spaziale e dopo aver delineato alcune limitazioni attuali di questo, l'A. expone brevemente, sulla seorta delle ricerche exeguite e delle ipotesi presentate da vari studiosi. le conoscenze sui più importanti problemi dellambiente di una cabina spaziale oecmpata dall uomo.

Vengono esaminati critieamente il rifomimento di $\mathrm{O}_{2}$ e l'eliminazione di $\mathrm{CO}_{2}$. Teliminazione del vapor daequa e delle altre sostanze gassose o volatili prodotte dall uomo o dalle apparecchiature della cabina, l'eliminazione ed il ricupero parziale o totale degli escereti, il mantenimento della pressione e della composizione dell aria o della miscela respirata. il mantenimento della temperatura.

Particolare considerazione viene data ai sistemi chinsi di rigenerazione dei gas respiratori. dei quali vengono discussi i diversi tipi in base allo stato attuale dei risultati sperimentali. alla possibiliti di pratiea attuazione, alla sicurezza ed al rendimento assoluto e relativo al tempo di impiego.

Si conchude che per viaggi di qualehe giorno sono ancora preferibili le seorte di $\mathrm{O}_{2}$ e la fissizione chimica of fision del $\mathrm{CO}_{2}$ e degli altri incuinanti. per viaggi di maggoior durata (settimane e mesi) appaiono attualmente migriori i cicli chinsi di rigenerazione chimica a fisico-chimica dell $\mathrm{O}_{2}$ dal $\mathrm{CO}_{2}$

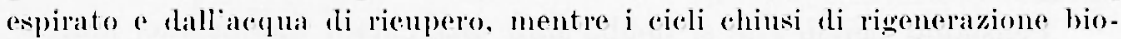
logica -.- peralto interessanti perehè atti a risolvere vari problemi contemporalneamente - presentano ancoral notevoli incognite.
\end{abstract}

SUMmarr. ... After a mention of the principal biologieal aspects of spaceflight and of a fow of its limitations. the duthor exposes briefly (on

$\left({ }^{*}\right)$ Lota presentata al $2^{\prime \prime}$ Congresso Internazionale Tecuico scientifico dello spazio. Roma. 19-23 Giugno 1962.

$(* *)$ Ispettorato di Sanita Aeronautica. Capo dell Ispettorato: Tent. Gen. med. C.S.A. Prof. T. Lomonaco.

centro di Studi e Riecrehe di Vedicina Aeronantica e spaziale. Di. rettore: Tent. Col. met. C.S.A. Prof. A. Samo. 
the basis of research performed and of hypotheses presented by various scientists) the existing knowledge on the most important problems of a space cahin occupied by man.

The supplying of $\mathrm{O}_{2}$ and the elimination of $\mathrm{CO}_{2}$, the elimination of steam and of other gaseons or volatile substances produced either by man or hy the cabin's equipment; the elimination and the partial or total recovery of excreta; the maintenance of pressure and of the composition of air or of the breathable mix; the maintenance of temperature. all of these elements are eritically examined.

special attention is devoted to the closed systems of regeneration of breathable gases. The main types of these are discussed on the hasis of the present state of experimental results. Also the possibility of practical application. security and yield (both absolute and relative to application times) are surveyed.

The conchusion is that $O_{2}$ supplies and chemical or physical fixing of $\mathrm{CO}_{2}$ and other defiling substances are preferable for journeys lasting a few days. On the other hand, for jomrneys in the order of weeks or months. closed cycles of chemical and physical-chemical regeneration of $\mathrm{O}_{2}$ and $\mathrm{CO}_{2}$ expired and of recovery water are to be preferred. Closed cycles of biological regeneration ... though interesting from the point of view of contemporary solution of several problems -. still present several unsolved questions.

Un illustre studioso e pioniere delle scienze aerospaziali, il von Karman, ha affermato qualche tempo fa: "Noi non siamo in grado di compiere viaggi verso gli altri sistemi solari, almeno in un futuro prevedibile: sono troppo distanti ".

Nella sua semplicità, questa frase ei sembra sintetizzare la posizione attuale della scienza nei confronti della cosmonautica propriamente detta, posizione non di sficlucia ver'so la possibilità prossime o lontane della ricerea in questo campo, ma di onesta e consapevole valutazione delle immani difficoltà che dovrà superare l'uomo se vorrà riuscire a navigare veramente tra i pianeti di altri sistemi solari.

Pensiamo perciò che si debba fare una chiara distinzione, almeno per quanto concerne il settore biologico, fra i problemi e gli studi concernenti il volo spaziale nell'ambito del nostro sistema solare, in parte risolti o affrontabili con le conoscenze delle quali disponiamo o possiamo mgionevolmente prevedere, ed i problemi della navigazione al di fuori di questo, i quali sono notevolnente più complessi ed in molti casi soltanto ipotizzati.

Vero i che il progresso in tutte le discipline, specialmente in quelle fisiche a tecnologiche, è così veloce, a volte con aperture impensabili, da sorpassare in breve tempo concetti e previsioni che apparivano quanto mai giustificati e razionali. 
ASPETTI BIOLOGTCI DEI VOLO SPAZIAIE

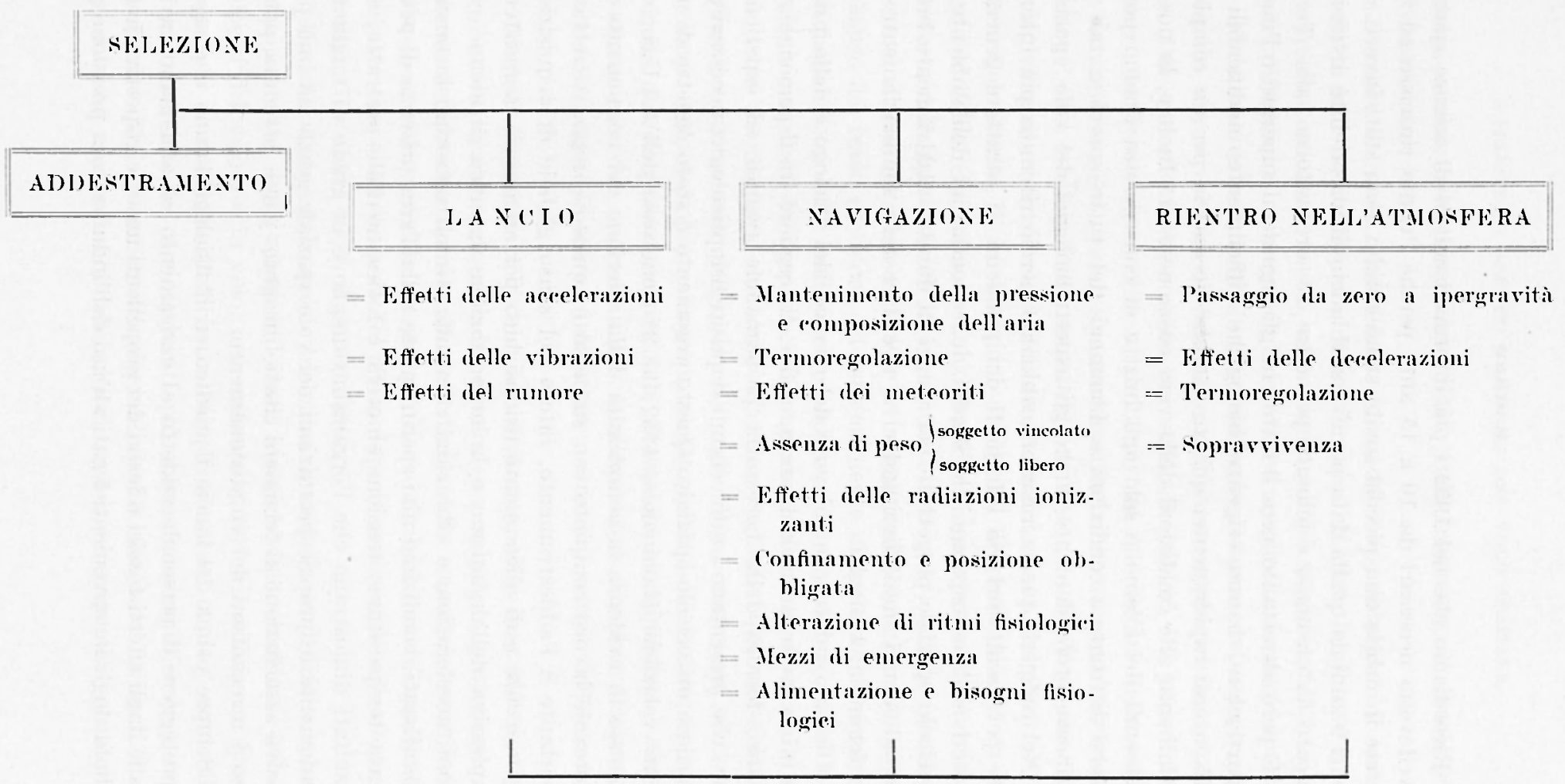

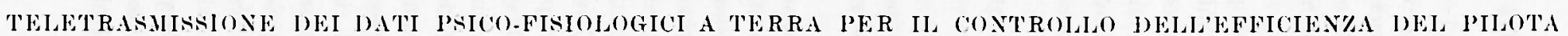


Ricordiamo che nel 1952 i più informati cultori di scienze sparziali prevedevano necessari da 10 a 15 anni perchè l'uomo riuscisse ad immettere in orbita una piceola sonda strumentale: sono stati invece sufficienti 9 anni da quella data per arersi il lancio di un satellite artificiale occupato da un nomo e pilotato per oltre 25 ore attomo alla Terra.

is però altrettanto vero il fatto che gli organismi superiori e l'uomo in particolare, hanno esigenze biologiche difficilmente modificabili di tanto, e cosi rapidamente, quanto sarebbe necessario per un completo adattamento alle condizioni della vera cosmonautica. Inoltre, la nostra conoscenza del cosmo è solo agli inizi e di conseguenza l'uomo potrà. trovarsi di fronte a condizioni e fenomeni del tutto nuovi e tali da modificare profondamente anche gli aspetti biologici del volo spaziale.

Nel redigere questa relazione abbiamo perciò ritenuto più interessante per ascoltatori già informati dei problemi di canttere gemerale, circoscrivere l'esposizione allo studio delle condizioni dell'ambiente in un veicolo spaziale progettato per viaggi di clumata relativamente breve (fino ad alcuni mesi) limitandoci a ricordare solo sommatramente gli altri fenomeni biologici.

Diciamo subito che il lavoro del medico, de fisiologo e dello psicologo inizia molto prima de] lancio, al fine di scegliere fra il personale volontario, fornito della necessaria prepalazione teconica ed esperienza, coloro che presentano i non commin requisiti fisiopsicologici neressari per' governare un veicolo spaziale. Questo argomento è stato oggetto di una recente relazione (Iomonaco, 1962) al $2^{\circ}$ Congresso dell'A.I.U.S., whe ha messo in evidenza la complessità della selezione del cosmomatuta ed il noterole lavoro compiuto con suecesso in questo campo. Altrettanto inportante ò l'addestmamento, inteso nel senso globale di acquisizione di conoscenze e di allenamento fisiopsichico per migliomare la resistenza complessiva dell'organismo e la sopportazione specifica di aleme condizioni: accelerazioni a vibrazioni rilevanti; stimoli sensoriali inconsueti e disturbanti; condizioni mai sperimentate sulla Terra (assenza di peso): elevata temperatum; tensione emotiva e fatica mentule protratte, ete. I risultati dimostrano che l'organismo umano è in grado di migliorare grandemente le proprie prestazioni nel volo spaziale grazie ad una progressiva assuefazione ai fenomeni citati, in gran parte ottemuta attraverso i meceanismi del condizionamento.

Sempre prima del lancio il medieo ed il fisiologo hamno il compito di proteggere il personale addetto al "aricamento ed alla manovia del missile dagli effetti tossici o lessivi dei propellenti usati, e rispettivamente il pilota dagli inconvenienti legati all'uso dell'indumento a pressione per 
il tempo, talora assai lungo fra l'inizio delle manove di lancio e la partenza.

Dumante il lancio, prina fase attiva del volo, dominano il quadro gli eftetti delle aceelerazioni e delle vibrazioni di varie lunghezze d'onda ed ampiezzal, sui quali non ai soffermiamo poiché l'appresentano (apitoli classici di medicina aeronaution trasferiti ed ampliati nel ampo spaziale.

Nella fase di volo orbitale od a notevole distanza dal campo gravitazionale terrestre si ha il fenomeno dell'assenza parziale o totale di peso. Dei suoi effetti e dei mezzi per studiarlo parlerà fra poco il Dott. Meineri. In questa fase, oltre ai problemi del microclima della cabina, che saranno esposti meno sommarianente nella seconda parte della relazione, ed a quelli dell'alimentazione, ne esistono altri, di origine psicofisiologico, concernenti il confinamento del cosmonauta, del quale parler'à il Ten. Col. Strollo, la sua posizione obbligata, la formazione di un equipaggio ed il suo rendimento in funzione delle singole personalità, dei turni di lavoro, della iperattività (come sinora è arvenuto) o della ipoattività (come si ritiene potrebbe avrenire in viagreri molto lunghi). Un aspetto biologico del tutto peculiare, ed estremamente importante, è rappresentato dagli effetti delle radiazioni cosmiche, ed in genere di tutte le radiazioni ionizzanti dello spazio, sull'organismo del cosmonatata. Di esso sarà detto ampiamente da altro relatore. Vogliamo qui solamente rirordare i considerevoli progressi che negli ultimi anni sono stati fatti nel campo della radioprotezione con sostanze ehimiche, tanto per lat prevenzione quanto per la terapia delle alterazioni biochimiche e cellulari, che damno motivo a sperare in una migliore difesa, sia pure temporanea (attraversamento di fasce radioattive, eruzioni solari) dell'organismo numano.

Durante il rientro nell'atmosfera terrestre, altra fase attiva, i problemi corrispondono a quelli del lancio, con in più la protezione dall'elevata temperatuma provocata dall'attrito della superficia esterna della cabina contro le molecole sempre più dense di aria, nonché quello della sopravvivenza e del ricupero del pilota dopo l'ammaraggio o l'atterraggio ron tutto il reicolo o con il paracadute.

Un lato di grande interesse biologico di tutta la navigazione spaziale è rappresentato dalla teletrasmissione e registrazione continue dei dati biologici del soggetto in rolo, non soltanto ai fini scientifici, ma specialmente per controllarine l'efficienza fisiopsichica così come ì controllato il funzionamento dei rari apparati più o meno automatici di governo del veicolo. Dai documentari cinematografici dei lanci orbitali statnnitensi, e dai rapporti scientifici che entro breve tempo sono stati messi 
a disposizione degli studiosi, appare chiaramente l'imponente organizzazione, che si avvale di un grandissimo numero di fisiologi e di medici, mediante la quale sono stati seguiti, praticamente senza soluzione di rontinuità, i principali fenomeni respiratori, circolatori, il comportamento e la parola del pilota. Ciò ha ronsentito, come è evirlente, non soltanto la sorveglianza del funzionamento del più perfetto, complesso e prezioso insieme di automatismi (ci si conceda il termine), installato nella capsula, ma ha anche permesso l'assistenza psicologica al pilota, altrettanto necessaria quanto una temperatura confortevole o l'apporto di cibo.

Delineati cosi, per necessità di tempo in morlo molto superficiale, i principali aspetti biologici del volo spaziale, possiamo trattare ora quelli dell'ambiente entro il quale deve vivere ed operare il cosmonauta.

Come è noto, le condizioni fisiche dello spazio differiscono profondamente da quelle esistenti negli strati più bassi dell'atmosfera terrestre e non consentono la sopravvivenza dell'uomo se non racchiuso entro cabine o scafandri a perfetta tenuta di gas, nei quali siano mantenuti valori di pressione, temperatura e composizione dei gas entro i limiti fisiologici.

Ciò comporta la soluzione di numerosi e complessi problemi di ordine biologico e tecnico, molti dei quali resi più difficili dagli altri requisiti del veicolo spaziale (limitazioni di peso e di posto, disponibilità di energia, etc.) e dalla navigazione in assenza di peso e con accelerazioni rilevanti. Per questo motivo, e per la considerevole mole di lavori originali, ancora non molto conosciuti, compiuti in questi ultimi anni, ci ì sembrato di qualche utilità trattarli con maggiore ampiezza in questa seconda parte della relazione.

Una elencazione dei principali probleni e dei metodi proposti e attuati per risolverli porta a considerare i seguenti argomenti:

1. - Rifornimento di $\mathrm{O}_{2}$;

2. - Eliminazione del $\mathrm{CO}_{2}$;

3. - Eliminazione del vapor d'acqua e di altre sostanze gassose e volatili prodotte dall'nomo o da apparec(hiature (batterie, fluidi idraulici o refrigeranti, etc.);

4. - Eliminazione e recupero degli escreti;

5. - Mantenimento della pressione e della composizione dell'aria;

6. - Mantenimento della temperatura.

Il rifornimento di $\mathrm{O}_{2}$ deve essere regolabile a seconda delle necessità dell'equipaggio e puo essere attuato mediante scorte di gas, composti 
chimici, con il ricupero ehimico o biologico del $\mathrm{CO}_{2}$, con la dissociazione elettrolitic'a dell' $\mathrm{H}_{2} \mathrm{O}$.

Le possibili sorgenti di $\mathrm{O}_{2}$, infatti, sono principalmente:

- il gas compresso in bombole (fino a $300 \mathrm{~atm}$ ), che ì la forma più maneggevole e idonea a fornire il gas alla portata necessaria: questo metodo comporta l'inconveniente del peso e dell'ingombro non indifferente delle bombole; è perciò utilizzabile solo per viaggi di breve durata;

- $\mathrm{l}^{\prime} \mathrm{O}_{2}$ liquido, che ha l'inconveniente di una evaporazione obbligatoria, non regolabile, che corrisponde nelle 24 ore a circa il $10 \%$ della massa di $\mathrm{O}_{2}$ trasportata. Ciò comporta l'esaurimento della scorta in breve tempo anche se il consumo è minimo.

Per rendere possibile l'impiego di $\mathrm{O}_{2}$ liquido quale scorta nel volo spaziale, Andrews (1961) e separatamente Hankins e Gardner (1960) hanno studiato convertitori in grado di funzionare anche in assenza di gravita, dimostratisi efficienti in varie posizioni ed a diversi valori gravitazionali: il campo di impiego è simile a quello del gas compresso;

- l'acqua: questa contiene l'88\% in peso di $\mathrm{O}_{2}$ ma la sua elettrolisi comporta un elevato consumo di energia elettrica (ca 14 Kwatt/h per $\mathrm{m}^{3}$ di $\left.\mathrm{O}_{2}\right)$. Con l'elettrolisi si possono ottenere cirea $500 \mathrm{~g}$ al giorno di $\mathrm{O}_{2}$ per $\mathrm{kg}$ di apparato;

- il perossido di idrogeno: si decompone spontaneamente con reazione esotermica e può fornire dal 50 al $90 \%$ in peso di $\mathrm{O}_{2}$, è però instabile e quindi diviene un pericoloso esplosivo; ha comunque il vantaggio di poter essere contenuto in recipienti leggeri a di forma non obbligata;

- il clorato di potassio $\mathrm{KClO}_{3}$, whe in presenza di biossido di manganese può fornire a il $40 \%$, in peso di $\mathrm{O}_{2}$ (la reazione non ì regolabile);

- i perossidi alcalini, particolarmente quello di potassio $\mathrm{K}_{2} \mathrm{O}_{4}$ il quale in presenza di aria umida si decompone lentamente con sviluppo di $\mathrm{O}$ e e formazione di $\mathrm{KOH}$ rhe, a sua volta, e utile perche fissa il co, dando luogo a $\mathrm{K}_{2} \mathrm{CO}_{3}$; il rendimento in peso di $\mathrm{O}_{2}$ ì del $33 \%$ e sono sufficienti circa $3,5 \mathrm{~kg}$ di perossido al giomo per 1 uomo; questo metodo ha un buon rendimento anche per periodi di diverse settimane;

- la dissociazione del $\mathrm{CO}_{2}$ con metodi fisici e fisico chimice à la soluzione più adeguata per viaggi di lunga durata e sarà trattata con 
maggiore ampiezza più avanti: occorre peró dire fin d'ora che questo metodo può fornire solo ca l's0\%, dell' $\mathrm{O}_{2}$ neecessario (in quanto il quoziente respiratorio medio dell'uomo a riposo o in lievissimo laroro muscolare ì intorno a quel valore) e che si frappongono ancoral numerose difficoltì teconiche perr il raggiungimento di un rendimento soddisfacente.

Ancle pere eliminare il (CO, esistono vari metodi, da quello classico dei fissatori chimicei ai più moderni che portano alla dissociazione della molecola ron ricupero dell $\mathrm{O}_{2}$. En'indagine sperimentale companativa dei fissatori chimici più afficacei è stata eseguita da noi (1958), portando alla conclusione (dhe i prodotti con rendimento più elevato sono l'idrossido di sodio (peraltro di difficile maneggio a deliquescente) e la monoetanolamina. Questa ultima è rigenemabile mediante riscaldamento e re risulta ora essere adoperata nei sottomarini atomici, ma necessita di una ulteriore filtrazione dell'aria per trattenere le trace di vapori ammoniacali che si sviluppano.

L'assorbimento da parte di zeoliti sintetiche non ai ha dato i risultati brillanti descritti da altri A $\mathrm{A}$; commonqe, la rigenerazione dei "setacei molecolari" di silico-alluminato di Na a Ca è difficile in quanto comporta il rapido trattamento termico di una massa cattiva conduttrice del calore per eliminare il $\mathrm{CO}_{2}$ assorbito.

Rerentemente Willard (1961) ha costruito un'apparecelhiatura rompleta (e notevolmente complessa) per la fissazione del $\mathrm{CO}_{2}$ con il setaceio molecolare Linde tijo $5-\Lambda$ e per la rigenerazione di questo, ma lo stesso A. sottolinea la necessità di ulteriori esperimenti per approfondire l'andamento di aleune variabili che influenzano il rendimento complessivo del sistema. Anche Boitean e Biget (1961) eonfermano la limitata efficacia di queste sostanze a propongono l'impiego di una miscela di LiOH e fibre di amianto, sotto forma di sottili discoidi, resistenti alle azioni mecaniche ed attivi fra 0 a 60 oc!. Interessante teoricamente lat diffusione selettiva del $\mathrm{CO}_{2}$, resa difficile in pratica dalla sua bassa concentrazione. Sulla fotolisi del $\mathrm{CO}_{2}$ e sulla riduzione mediante $\mathrm{H}$, si avrà oceasione di tornare fra breve.

Il problema della eliminazione dell'anidride arbonica è però solo uno degli aspetti della depurazione dell'aria di un ambiente confinato: 
la presenza dell'uono comporta infatti lacenumbo progressivo di altre sostanze volatili e gassose provenienti dalla cute (particolaremente nella sudorazione), dall'intestino e dalla superficie alveolare. Sono state individuate sostanze organiche quali gri acidi capronico, caprilico, valerianico, butirrico, acetico, lattico, formico e l'acroleina, tutte di provenienza cutanea; lo scatolo, l'indolo, il metano, l'acido solfidrico, di prorenienza intestimale; $\mathrm{l}^{\prime} \mathrm{NH}_{3}$, alcooli, etce, presenti in trace ninime nellaria espirata. Ia produzione di molto fra esse è facilitata dalle fermentazioni dei detriti cutanei, alimentari e del sudore che arvengono soprattutto per azione di germi quando non i curata la pulizia della pelle, del aro orale e degli indumenti (come probabilmente arverrà in individui confinati in mo spazio molto limitato e con scorte di acqua per uso esclusivamente alimentare).

Le sostanze sopra elencate hamno mal scarsa tossicità alle concentrazioni che si osservano negli ambienti male aerati, possono peró prorocare disturbi considerevoli negli individui viventi in un abitacolo stagno, rome is dimostrato dall'esperienza fatta nei sommergibili.

Il McFarland (1946), che sottopone ad una critica severa il loro possibile effetto dammoso, ammette che esse possono causare disturbi (difficolti a srolgere attività fisioa, astenia, nausea, romito, cefalea, etc.) e sottolinea l'aggravamento causato da eventuale mal d'aria con vomito e dialrea in qualcuno dei soggetti.

Anche le abnormi fermentazioni intestinali provocano ammento nella concentrazione di aleme fra la dette sostanze. Bisogna inolte tener presente la possibile produzione di vapori o di gas dannosi da parte delle batterie elettriche, degli impianti idmalici di rafireddamento e - nel caso di abine per lunghe navigazioni - dalla cucina. Gli eftetti della respilazione protratta di vari gas e vapori in concentrazione non tossica, alcuni dei quali potrebbero essere presenti in concentrazioni analoghe nell'aria di un ambiente ronfinato oceupato da soggetti umani, sono stati di recente studiati sperimentalmente da Sandage (1961). Questi ha esposto animali di diversa specie, per 90 giomi alla respirazione continma di aria contenente $20 \mathrm{ppm}$ di $\mathrm{H}_{2} \mathrm{~S}, 50 \mathrm{ppm}$ di metil mereaptano, $10,5 \mathrm{ppm}$ di indolo e 3,5 di scatolo, concentrazioni che lapporesentano le massime consentite in CSA per esposizioni intermittenti di tipo inclustriale. Al termine del periodo stabilito, l'A. ha trovato un aumento apprezzabile di solfoemoglobina nei ratti e nelle scimmie, un aumento noterole nel topino, sensibile grado di emolisi, alterazioni polnonuri (selo nel topino), elevata mortalita $(80 \%)$ delle scimmie e dei topini. Invece, il fenolo alla corecentrazione di s̆ ppon fu tollemato senza inconvenienti. 
Tor concentrazioni massine di altri gats e vapori tollewabili per lungo periodo di tempo dall'uomo sono le seguenti (Keating, 1959):

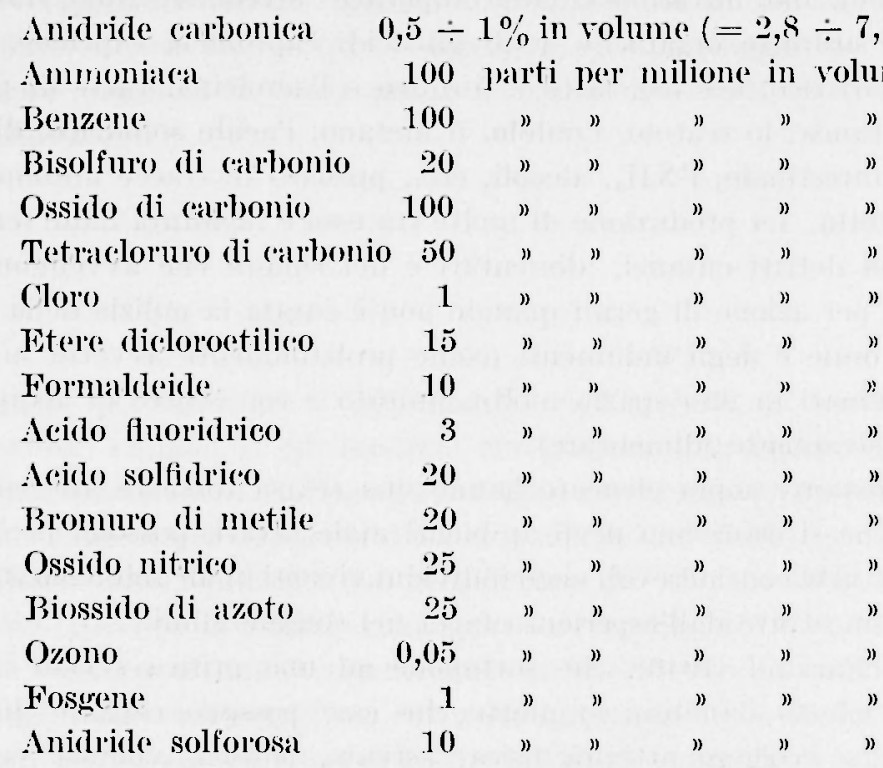

Per il progetto Apollo (eabina spaziale che dovrà mettere in orbita 3 uomini intorno alla luna) è stato costruito un "bruciatore di sostanze tossiche " di $4 \mathrm{~kg}$ di peso nel quale, mediante ossidazione catalitica e filtrazione, si eliminano tutte le sostanze inquinanti.

Al fissaggio del CO, può seguire il ricupero dell'O, come nel metodo proposto da Shearer e coll. (1962): il $\mathrm{CO}_{2}$ espirato è adsorbito da uno strato di setacei molecolari e, cosi concentrato, agisce su ossido di litio:

$\mathrm{CO}_{2}+\mathrm{Ti}_{0} \mathrm{O} \longrightarrow \mathrm{Ti}_{2} \mathrm{CO}_{3}$. Il carbonato fuso è sottoposto ad elettrolisi fornendo di nuovo ossido, arbonio ed $\mathrm{O}_{2}$ :

$$
\mathrm{Li}_{2} \mathrm{CO}_{3} \longrightarrow \mathrm{Li}_{n} \mathrm{O}+\mathrm{C}+\mathrm{O}_{2} \text {. }
$$

i stato cosi oftenuto un gas puro al $99 \%$, contenente ca l'1\% di $\mathrm{CO}_{2}$ e ca 200 ppm di $\mathrm{CO}$, quest ultimo facilmente ossidato con un adatto catalizzatore. Dallo studio analitico di questi ricercatori risalta che occorrono a 800 watt di energitı elettrica per fornire l'O, necessurio ad un nomo, con un peso di apparecehiatura di ca $110 \mathrm{~kg}$ e vari inconvenienti, fra i quali la necessità di trattare gas secchi perchè la presenza di vapore acqueo diminuisce il rendimento dell'elettrolisi.

Il potassio perossido $\mathrm{KO},\left(0 \mathrm{~K}_{2} \mathrm{O}_{4}\right.$ ) rappresenta, secondo le ricerche di Keating e Weiswurm (1960) un composto assai vantaggioso per la 
rigenerazione dell'aria. Esso ha infatti non pochi requisiti favorevoli: minimo peso ed ingombro; indipendenza da sorgenti esterne di energia; assenza di manutenzione o di parti in moto; capacità di provocare una sufficiente circolazione di aria esereitando un'attanaione chimica sul vapor d'acqua e sul $\mathrm{CO}_{2}$; azione purificante sui contaminanti chimici e batterici dell'atmosfera a causa del suo energico potere ossidante.

Le reazioni chimiche di questa sostanza con l'aria non sono perfettamente conosciute. Secondo gli $A \Lambda$. citati le più probabili sono le seguenti:
1) $2 \mathrm{KO}_{2}+\mathrm{H}_{2} \mathrm{O}=2 \mathrm{KOH}+3 / 2 \mathrm{O}_{2}$
2) $2 \mathrm{KO}+\mathrm{CO}_{2}=\mathrm{K}_{2} \mathrm{CO}_{3}+3 / 2 \mathrm{O}_{2}$
3) $2 \mathrm{KO}+\mathrm{CO}=\mathrm{K}_{2} \mathrm{CO}_{3}+\mathrm{O}_{2}$
4) $2 \mathrm{KOH}+\mathrm{CO}_{2}=\mathrm{K}_{2} \mathrm{CO}_{3}+\mathrm{H}_{2} \mathrm{O}$
5) $\mathrm{KOH}+\mathrm{CO}_{2}=\mathrm{KHCO}_{3}$
(i) $t / 3 \mathrm{KOH}+\mathrm{H}_{2} \mathrm{O}=t / 3\left(\mathrm{KOH} \cdot 3 / 4 \mathrm{H}_{2} \mathrm{O}\right)$
7) $\mathrm{KOH}+\mathrm{H}_{2} \mathrm{O}=\mathrm{KOH} \cdot \mathrm{H}_{2} \mathrm{O}$
8) $1 / 2 \mathrm{KOH}+\mathrm{H}_{2} \mathrm{O}=12\left(\mathrm{KOH} \cdot 2 \mathrm{H}_{2} \mathrm{O}\right)$
9) $2 \mathrm{~K}_{2} \mathrm{CO}_{3}+\mathrm{H}_{2} \mathrm{O}=2\left(\mathrm{~K}_{2} \mathrm{CO}_{3} \cdot 1 / 2 \mathrm{H}_{2} \mathrm{O}\right)$
10) $2 / 3 \mathrm{~K}_{2} \mathrm{CO}_{3}+\mathrm{H}_{2} \mathrm{O}=2 / 3\left(\mathrm{~K}_{2} \mathrm{CO}_{3} \cdot 3 / 2 \mathrm{H}_{2} \mathrm{O}\right)$

Ie reazioni sono nel complesso esotermiche ed il calore prodotto deve essere rapidamente disperso, altrimenti si raggiunge la temperatuma di ignizione nella massa chimica con liberazione di grandi quantità di $\mathrm{O}_{2}$; anche per questo motivo i granuli di $\mathrm{KO}_{2}$ devono essere disposti in uno strato dello spessore delle singole unità, che offre la massima superficie all'aria ed i contenitori devono essere esposti uno dopo l'altro man mano che si esauriscono. La polvere che proviene da questi è estremamente caustica e perciò è necessario indossare una maschera antipolvere, occhiali e gnanti ed attendere, per togliere la copertura di lana di vetro del contenitore, che la reazione sia iniziata su tutta la superficie del reagente.

Le prove fatte (una delle quali durata 7 giomi) hamo dimostrato il buon rendimento pratico di questo metodo, senza l'inconveniente teoricamente preverdibile di una sovraproduzione di $O_{2}$. Con poco più di $150 \mathrm{~g}$ di $\mathrm{KO}_{2}$ per $\mathrm{nomo} / \mathrm{h}$ è stata ottenuta m'aria con il $20 \div 25 \% \mathrm{O}_{2}$, $0,5 \% \mathrm{CO}_{2}, 60 \div 50 \%$ di umidità relativa, priva di odori e contaminanti. Il consumo è alquanto superiore nelle prove di lunga durata.

L'eliminazione del vapor d'acqua dall'ambiente non presenta difficoltà rilevanti e, se avviene con regolarità, contribuisce anche al mantenimento di una conforterole temperatuma efficace. Dal resoconto della 
prima ascensione in pallone oltre i 30.000 metri (Simons, 1957) lisulta ('he l'umidità relativa dentro l'abitacolo presentio un incremento non previsto, che aggravo le conseguenze dell'aumento di temperatura calusato dallimperfetto funzionamento del sistema refrigerante.

Il vapore acqueo puo essere trattenuto con varie sostanze chimiche solicke (cloruro di calcio, solfato di calcio o di sodio, silica-gel, ossido di bario o di calcio, cloruro di litio, zeoliti sintetiche, idrossido di potassio o di sodio) e liquide (glicerina, acido solforico, alcooli). Sono da preferire quelle più facilmente maneggiabili e che si possono rigenerare con il calore, quali le zeoliti sintetiche e setacei molecolari, ed il gel di silice. Ugualmente raceomandabili sono i metodi fisiei (condensazione per raffreddamento, compressione). In ambedue i casi, volendo ntilizzare laacqua ricuperata, occorre purificarla filtrandola attraverso carboni attivati o

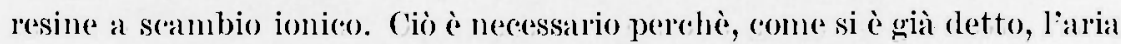
contiene un certo numero di sostanze inquinanti e di odori che possono ('ausare disturbi non indifferenti all'equipaggio.

Il ricupero dell'ar'qua dai rifiuti e l'utilizzazione o la conservazione della parte restante ì stato affrontato sul piano teorico ed applicativo da vari autori. Kefl, Bambenek e I)es Jardins (1959 a 1960) hammo eseguito ricerche considerando le esigenzo di viagegi spaziali di media durata (da alcuni giomi a 12 mesi) e di un equiparguio limitato (2-10 nomini). Poiche l'uomo introduce in media con le bevande a gli alimenti ca 2200 or di acqua al giomo - alquanto di più secondo altri $\Lambda \mathrm{A}$ - e ne elinina $2500(300 \mathrm{~g}$ provengono dalle reazioni di ossidazione degli alimenti) i sufficiente che il sistema di rieupero abbia un rendimento intorno al $90 \%$. Somo stati esperimentati, impiegando urine oppure urine e feci, i seguenti metodi: distillazione (risultati migliori con la distillazione nel vuoto, a bassa temperatura, di sola urina); congelamento nel vuoto o liofilizzazione, che ha un rendimento del $96_{\%}^{\circ}$ usando solo urine (si ha sublimazione di cristalli di ghiaceio che forniscono acqua leggemente torbida e con batteri, per cui sono necessarie la filtazione e la potabilizzazione (ohimioa); refrigerazione (basso rendimento); elettrodialisi (residua una notevole quantità di elettroliti e di sostanze organiche per i quali oceore una ulteriore depurazione): filtrazione e scambio di ioni (rappresentano di regola metodi sussidiari ai precedenti). Gli $A A$ ritati concludono rhe $i$ metodi migliori per rendimento, modico peso e volume, sono la distillazione sotto vuoto associata all'uso di resine a scambio ionico e di carbone attivato per elininare l'odore ammoniacale ed altre impurità. Essi ritengono inoltre che l'urina debba essere laceolta e trattata separatamente dalle feci e a tal fine descrivono vari espedienti di raccolta. 
Per quanto riguarda l'urina, quest'ultimo problema era sorto già con l'introduzione nell'esereizio del rolo degli indumenti a pressione. Rerleden (1961) la descritto un ralecoglitore di gomma che viene portato in permanenza, sostenuto da una cintuma e fornito di una valvola posta all'esterno dell'indumento, che permette il trisferimento dell'urina in un apposito saceo di polivinile.

E previsto l'inpiego delle feed disidratate rome sostanze nutritive per le alghe nel "aso di riclo biologico ehiuso ed anche come scherno contro le radiazioni in sostituzione di quello rappresentato dalle scorte di alimenti disidläati consumati dumante il viaggero.

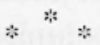

Il mantenimento di una sufficiente pressione entro la cabina, assolutamente necessaria per la vita dell'uomo, è un problema di ordine tecolologico tuftora non perfettamente pisolto nel senso che non si it riusciti ad impedire la fuga continua di piccole quantita di gass att lälverso minime fessure. Ad esempio, nella pianificazione del rifornimento di $\mathrm{O}_{2}$ per la capesula Merceury è stato previsto un ronsumo metabolico di 500 ce/min, più che sufficiente alla lieve attività tisiea del pilota, ed una perelita dalla cabina di $300 \mathrm{cc} / \mathrm{min}$, con possibilita di compenso fino a 2500 es/min. E probabile che materiali mighliori e l'eliminazione di ogni diseontinuita non indispensabile nelle pareti della cabina possano diminuire queste perdite. Va tenuta presente anche la possibilità di fori prodotti dall'impatto di piceoli meteoriti, nei cui confronti potrebbe essere utile l'impiego di una intereapedine di sostanze plastiche che, divariente da un proiettile, riprendono la loro forma oceludendo il foro cosi provocito.

Un mezzo per diminuire l'entità delle perdite è rappresentato dalla diminuzione del gratiente di pressione fra cabina ed ambiente esterno. Nella ritata capsula Mereury la pressione è stata mantenuta a cal 350 numHg, con respilazione di $\mathrm{O}_{2}$.

L'atmosfera della cabina non dovrebbe peró essere costituita da $\mathrm{O}_{2}$ puro anche se a pressione parziale fisiologica: varie ragioni infatti consigliano la presenza di un gas inerte nella miscela respirata dal pilota spaziale: la respirazione di $O$, puro per molto tempo provora facilnente atelettasia polmonare nelle zone ipoventilate a causa della mapida diffusione dell'O, attarareso la parete alveolate e dell'assenza di wn gas inerte che ne ocema la cavita. Un femomeno amalogo, che si manifesta ron dolore, accade a carico dellorecehio medio e delle carità pamanasali 
nel caso di occlusione dei tragitti di comunicazione con naso e faringe (secondo Comroe e Dripps, 1950 , arviene nel $25 \%$ dei soggetti sottoposti ad ossigenoterapia).

Un pericolo peculiare è lappresentato dall'attraversamento della cabina spaziale da parte di piccoli meteoriti animati da elevata velocità: questi provocano una "esplosione vaporizzante " tanto più grave quanto maggiore la concentrazione di $\mathrm{O}_{2}$ presente nell'aria (Gell, 1961). Non sembra raccomandabile l'impiego dell'elio come gas inerte al posto dell'azoto poiché, a causa della sua minima densità, può sfuggire più facilmente attraverso piccole imperfezioni di tenuta della cabina. La presenza di azoto è raccomandata anche per diminuire le probabilità di incendio, ma rappresenta una notevole complicazione tecnica poiché, a causa delle finora inevitabili perdite delle cabine stagne, occorre sostituire l'azoto sfuggito con uguali volumi provenienti da una adatto serbatoio, possibilmente in maniera automatica.

Dai resoconti dei voli spaziali dei Vostok risulta che la pressione in cabina era di 1 atmosfera e la pressione parziale di $\mathrm{O}_{2}$ intorno a quella di livello del mare.

La temperatura dentro una cabina spaziale dipende essenzialmente dalla temperatura e dalle altre caratteristiche fisiche extra-atmosferiche, dal potere isolante delle pareti e dalle qualità delle loro superfici esterna ed interna, dalle sorgenti di calore presenti nella cabina (comprese quelle di natura biologica) ed infine dalla sottmazione di calore da parte di fenomeni endotermici di natura chimica o fisica che si svolgono al suo interno. Le ricerche di fisica hamno dimostrato un progressivo e rilevante aumento della temperatura al di fuori dell'atmosfera terrestre con inizio intorno agli $80 \mathrm{~km}$ per oltrepassare 1000 oC oltre i $400 \mathrm{~km}$.

Gli effetti fisici e biologici di questa temperatura cosi elevata sono profondamente diversi rispetto a quelli noti per i corpi entro l'atmosfera terrestre. Ciò dipende dal fatto che l'estrema rarefazione degli atomi gassosi a quelle altitudini impedisce praticamente qualsiasi scambio di calore per convezione e che la quantità di calore ceduta dai rari atomi (he vengono a contatto con le pareti della cabina è infinitesima: in altri termini, pur essendo molto elevata l'energia cinetica di queste particelle, la loro massa è trascurabile e di conseguenza la quantità di calore presente nell'ambiente spaziale è insignifieante. La cabina quindi potrà ricevere 0 cedere calore nello spazio solo con il mexcanismo dell'irradiazione: le parti colpite dai raggi solari si riscalderanno, e quelle in ombra si raffredderanno, in misura dipendente dal gradiente di temperatura e dalle caratteristiche del rivestimento esterno: una superficie levigata, bianca, 
speculare rifletterà la massima parte dei raggi ed assorbirà o cederà pochissimo calore; una superficie opaca, nera, assorbirà o cederà la quasi totalità del calore. Orientando opportumamente rispetto al sole le pareti della cabina fornite di caratteristiche assorbenti o riflettenti si può utilizzare entro i limiti necessari l'energia termica del sole. Questa può rappresentare inoltre una importante sorgente di energia per le varie apparechiature, sia che venga utilizzata direttamente come calore assorbito da corpi neri, sia che venga trasformata in corrente clettrica da batterie di fotocellule o da elementi temoelettrici (la differenza di temperatura fra lato al sole e lato in ombra sarebbe di a 300 oC).

In effetti, la quantità di calore prodotta entro la cabina è tale da richiederne la dispersione piuttosto che l'assumzione per mantenere nel suo interno una temperatura confortevole per l'uomo.

Allo scopo di disperdere l'eccesso di calore occorrerà disporre di ladiatori, che dovianno essere orientati verso il cielo (con temperatura efficace intorno ai $4^{\circ} \mathrm{K}$ ); meno conveniente è l'orientamento verso la terra, che presenta una temperatura media di ca $278^{\circ} \mathrm{K}$ e meno ancola verso il sole. Tenuto conto che la produzione di calore in ma cabina per singolo occupante puó andare da ca $500 \mathrm{~K}$ (al/h nei primi tipi a 1000 Keal/h nei modelli più recenti (alore prodotto in parte dall'organismo ed in parte magriore dalle apparecchiature elettroniche e meconiche), Jacobson (1960) calcola che la semplice dispersione attraverso una parete della superficie della cabina non è sufficiente e che occorre un sistema di raffreddamento; dei vari possibili, offre le calatteristiche più idonese il ciclo a vapore acqueo. Nella capsula Mercury è stato installato uno scambiatore di calore per la termoregolazione dell'aria della cabina ed un secondo per quella dell'indumento pressurizzato. La superficie metallica esterna della stessa capsula è ondulata, verosimilmente per aumentarne l'area radiante.

Una condizione speciale è rappresentata dal notevolissimo aumento della temperatura sulla superficie estema della cabina dumante il rientro nell'atmosfera terrestre, a causa dell'attrito con le molecole di aria sempre più densa. Sono state escogitate protezioni di vario tipo, consistenti nel ricoprire le parti rhe penetrano nell'atmosfera con sostanze la cui fusione sottrae calore, con schermi di berillio, ete. Si ì naturalmente curato l'isolamento termico delle pareti. Dal punto di vista fisiologico è utile la diminuzione dell'umidità relativa nella cabina e dentro l'indumento a pressione nonché un adeguato pre-raffreddamento del corpo del pilota, ottenibile con la diminuzione della temperatuma dell'aria circolante entro l'indumento ventilato indossato sotto quello a pressione. Tutti i 
resoconti del rienteo da roli orbitali depongono tuttavia per un rilevante aumento di temperatuma nella abina ed entro loindumento, ed in qualehe raso anche per una transitoria ma seonforterole ipertemia del pilota.

$$
\therefore \quad \therefore
$$

Esaminati per sommi (appi i principali aspetti del microclima di un veicolo spaziale, vorremmo ora esporre le conoseenze attuali sni cosicldetti sistemi a ciclo respilatorio chiuso in quanto essi rappresentano la soluzione piò razionale per la rigenesazione dell'aria mediante apporto di sola energia e senza la necessità di scorte di gas o di sostanze chimiche con peso e volume proibitivi. In effecti, prima di giungere a questo concetto relativamente limitato, sono state avamate suggestive proposte per la costruzione di sistemi ecologici chiusi entro la cabina, introducendovi colture di piante in equilibrio biologico con gli animali.

Quest'idlea è stata preconizzata da Ross (1947) che propose l'impiego di piante verdi per la rigenerazione dellatmosfera, ed è stata posta sul piano sperimentale da Strughold e dai suoi collaboratori Phillips (1952) a Myers (1954), oltre che da ricereatori di altri laboratori (Bowman, 1953). I risultati di queste e di valie altre indagini sucessive, hanno dimostrato la possibilità di ottenere in laboratorio colture di alghe redi con un'elevata produzione di $\mathrm{O}_{2}$ fotosintetica ed in grado di fornite la quantità sufficiente ad un piccolo mammifero per molte settimane.

Recenti conferme somo riportate da Benoit e Coll. (1960), i quali espongono uno studio approfondito sulle varie specie vegetali esplorate a questo fine e sui requisiti neceessaldi per un sistema erologien rehiuso (entità della produzione di $\mathrm{O}_{2}$, strettamente lesata alla velocita di acecrescimento; rontinuità di produzione, dipendente dalla stabilità delle coltura; valore nutritivo della pianta; capacità di sviluppare utilizzando i rifiuti dell'nomo). Anche Bates (1961) riporta successi notevoli ottenuti con questo metodo: ad esempio, attraverso un'oculata scelta di alghe si sono trovati ceppe (come quello termofilo di Chloredla pyenoidosa o la specie Gonzales di synerhocystis) i quali hanno un'alta produzione di $\mathrm{O}_{2}$, con un rapporto di $\mathrm{CO}_{2}$ assorbito / $\mathrm{O}_{2}$ proclotto che può essere portato ron speciali espedienti a 0,8 , sopportano tempelature relativamente clevate, utilizzano parte degli escreti umani, non sono sensibili agli antibiotici necessari pere inibire l'acerescimento di batteri nella (coltum, atc.

Tali suceessi non devono peró far dimenticare rhe sono stati ottenuti in labonatorio, rioè in endizioni estremamente favorevoli per 
quanto riguarda la neecessità di nutrizione, accrescimento, riproduzione e difesa delle alghe impiegate. Ie colture di alghe abbisognamo, infatti, non soltanto di composti azotati in misura superiore a quanto normalmente eliminato dall'uomo, ma anche di in gran numero di elementi (in quantità rarie), la cui concentrazione nel liquido nut ritivo deve oseillare entro limiti ristretti, di un equilibrio acidi-basi prossimo alla nentralità, di una illuminazione costante a di determinata lunghezza d'onda, del regolare trapianto in soluzione fresca, dellassenza di germi o cataboliti inibitori dell'acerescimento, ete.;

Imprevedibili alterazioni genetiche da moliazioni ionizzanti possono dar luogo a mutanti inadatte alle condizioni obbligate dallo scambiatore di gas. Inoltre il peso ed il rolume delle colture e apparati sussidiari e lenergia oceorrente al mantenimento del processo fotosintetico sono notevoli: si parla di 500 kg per fornire l'(), neecessario ad un uomo e di 10 Kwatt per l'illuminazione giomaliema. A nostro arviso, gli aspetti piò discutibili sono rappresentati dal perpeolo della cessazione della funzione fotosintetica per inaleguata illuminazione della coltura, con invasione dellambiente da parte del $\mathrm{CO}_{2}$ metabolico dellaalga e dalla instabilità di questa pere cui è possibile la morte in massa della coltura in breve tempo. it indubbio che il sistema ecologico chiuso meriti ulteriori studi per il superamento di queste clifficoltà, anche perehè risolve (ontemporaneamente (almeno in parte) il problema della alimentazione nei viaggi di lunga dumta, ma per il momento non pare di prossima attuazione nei reicoli spazi:li.

('i sembrano pereio di noterole interesse i tentativi concreti che si fanno da qualche anmo per applicalre, migliorando il remdimento, alcume reazioni note di riduzione di $\mathrm{CO}_{2}$, per via chimier o fisica-chimiea ai sistemi di rigenerazione dell'aria. Utile testimonianza di questo orientamento è un Simposio sui sistemi a ciclo respiratorio chinso, tenutos $\mathrm{i}$ nell Aprile del 1960 presso la Mright Air Derelopment Division, dai cui Atti traiamo le notizie che segruono. Trascumando i procedimenti che necessitano di temperature eceessivamente elevate (decomposizione termica) o che hanno un rendimento inaceettabile, il ricupero dello o, si puo oftenere essenzialmente in due modi. Il primo è moppresentato clalla reazione di Sabatier (1902):

$$
\mathrm{CO}_{2}+4 \mathrm{H}_{2} \longrightarrow\left(\mathrm{HI}_{4}+2 \mathrm{H}_{2} \mathrm{O}\right)
$$

la quale si svolge alla temperatura di 200 oc in presenza di un ataliz-

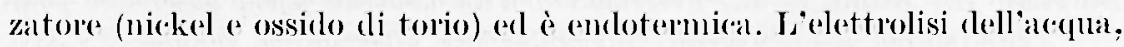
ben nota, permette l'ottenimento di O. gassoso e dell'H, neecesiario alla 
piduzione del $\mathrm{CO}_{2}$. Thale a Tamplin (1960) ahe hamno sperimentato questo matodo, lo ritengono raccomandabile perché possiede molti dei requisiti necessari nella cabina spaziale: non è disturbato dalle aceelerazioni a dell'assenza di peso, occupa poco rolume, non richiede molta energia elettrica, funziona automaticamente con minima manutenzione e si puo regolare a seconda del bisogno, non si esaurisce, non è sensibile a variazioni di temperature ambientale ed alle radiazioni. Il suo attuale rendimento puó essere migliorato.

Il secondo metodo consiste nella liduzione diretta, in presenza di atalizzatore, del $\mathrm{CO}_{2}$ a grafite: $\mathrm{CO}_{2}+2 \mathrm{H}_{2} \underset{\mathrm{C}}{\longrightarrow}+2 \mathrm{H}_{2} \mathrm{O}$.

Foster e Mc.Nulty (1961) l'hanno sagroiato sperimentalmente, impiegando il Fe come catalizzatore ed hanno ottenuto, per effetto della riduzione di 30 litri di $\mathrm{CO}_{2}$ in 1 ola, $16 \mathrm{~g}$ di rarbonio solido. Fssi hamno studiato le migliori condizioni di temperatura, pressione, caratteri dei gramuli di ferro, i mezzi per separare l'acpua formatasi, la rigenerazione del catalizzatore ed hanno costruito un modello di apparecechio. A seguito di queste ricerche hanno realizzato un apparecehio di tipo industriale, capace di ridure soo ce/min di CO, tale apparato, poco ingombrante e di peso modesto, si è dimostrato in grado di "lavorape "l'anidride prodotta da un uomo durante tre gromi, senza abbisognare praticamente di manutenzione.

11 raffronto fra il peso delle scorte di $\mathrm{O}_{2}$ a depuratori, quello di un apparato per il pireupero chimico dell'O, e dell'acqua e quello infine di un apparato per il recupero biologico dimostra che il secondo è il più conveniente per viaggi superiori ad uma settimana e fino ald oltre tre anni, cioc si presta a soddisfare le esigenze degli scambi gassosi di un piceolo equipaggio previsto per l'esplomazone entro l'ambito del sistema solare.

Allo stato attuale delle conoscenze questa via appare quindi la più realistical a sicura.

\section{BIBIAOCRAFIA}

Axwnws R. C., Zero-gravit! liquid oxygen comerter. "AsD Tech. Rep. 61-431", (sept. 1961).

Bates J. II., Recent aspects in the dexelopment of a closent ecologie system. "Aerosp. Med, ", 32, 12, (1961).

BexotT R. J., Traisek l'., Bacecki A., Selection of an alga for a pholosymlietic gas erchnuger. "WADD Tech. Rep. 60-163", (Feb. 1960.)

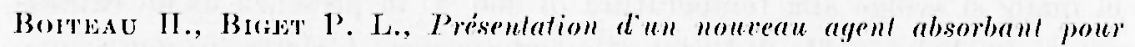
l'climinalion de l'anhydride carbonique des almospheres confinees, "Atti Congr. Intern. Mérl. Acron. Cosmon. "), Paris, Sept. 1961, 
Bownax X. I., The food and atmospherie control problem on space eressels. I. Chemical purification of air. II. The use of algae for food and atmosphere comtrol. "J. Brit. Interplan. Soc. ", 12, II8 e 159, (1953).

Calvis M., Pholosymthesis, in "Radiation Biology and Vedieine ", Mhdison Wesley Co., Readling, Massachussets, (1958).

Closed cirenit respiratory system symmosium. "WADD) Tech. Rep. 60-5it", (Alug. 1960).

Cozza A., Prevenzione chimira delle lesioni da radiaziomi iomizzanti, "2" Corso Internaz. Uff. Med. ", Firenze, ti-16 Giugno 1962.

DALE s. H., TAMPLA A. R., The Sabatier reaction for inorganic recocery of orygen in manned space capsule, in "WADD l'eeh. Rep. 60-57t"

Des JaRdiss J., ZeFF .I. D), Banbexick R. A., Waste collection unit for a space rehicle. "WADD Tech. Rep. 60-290", (May 1960).

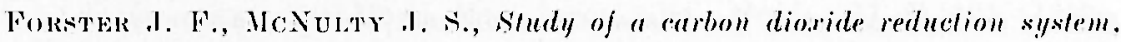
"Asl) Tech. Rep. 61-38s", (Alug. 1961).

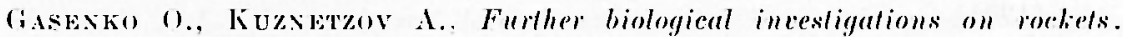
"Atti 2" Congr. Mondiale Med. Aeron. Spaz", Roma (1959).

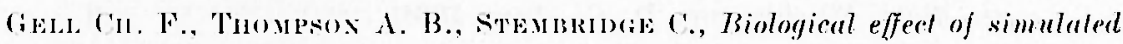
micrometeoroid penetration of a sealed chamber containing animal sperrimens. "Aerospace Medicine ", 33, 156, (1962).

Ginlakn R., BADRE R.. Problèmes almospliériques posés par les sous-marins a propulsion mucléaire. "Rev. Corps Santi Armeses", 2. 485. (1961).

Gurfisket, V. S., Isakoy P. K., Malkix V. B., Popoy V. I., La coordinazione della possura e dri movimenti dell uomo in condizioni di grarifazione aumentala e ridoffa. "Bjull. Eksiper. Biol. i Medits", 11. 12, (1959).

Gorriax D. A.. Eeffects biologiques des radialions cosmiquess sur les Spoutnilis. "C. R. Xe Congrès Eur. Méd. Aéron Cosmon. ". Paris, (196L).

Inasy 'T'. J., Chemical protertion against three types of radiation death, namely haemopoietice gastroinlestinal and the central neroous system. " 2 " Simposio dell A.I.U.A. ", Milano. 20 Aprile 1962.

IIAMMER L. II., Aeronantical system division studies in weighllessmess: 19591960. "WADD Tech. Rep. 60-715", (1)ec. 1961).

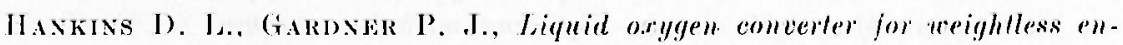
rironment. "AsI) Tech. Rep, 61-634", (Nov. 1961).

Jacossox S. L., Engineering of the sealed cabin almosphere control system. "Aerospace Medicine", 31. 388. (1960).

Kistrixa D. A.. Design parameters for the engineering of closed respiratory syskems. "WAD)' Tech. Rep. 59-766", (Dec. 1959).

Kentixa: D). A., Rounde R. W., (losed ecology. "WADD) Terh. Rep. 61-129", (IIarch 1961).

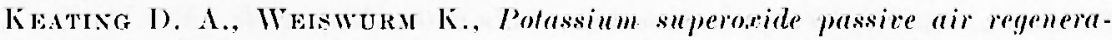
fion studies jor manned sealed enrironments. "WADD Tech. Rep. G0$707 \%$, (I)ec. 1960$)$. 


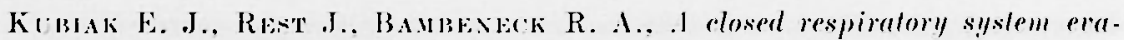
luralor. "ASI) Teech. Rep. 61-512" (Nept. 1961).

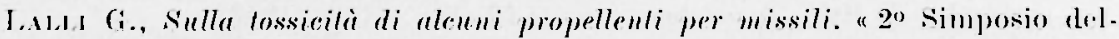
l'A.I.l.s. ". Vilano, 20 Aprile 1962.

Lonosaco T., Previsioni sul comportamento psieofisiologico dell uomo lancialo tello spezio. "Min. Med. ", 50. 1122. (1959).

lonoxaco T., Lineamenli di una selezione psico-fisien del cosmonaula. "2o Simposio dell A.I.l.s. n, Nilano, 20 Aprile 1962.

Ionoxaco T., Soxo A., Gili eljelli fisiopalologici del rolo spaziale ed i pro. blemi biologici della cosmonantiea. "Llisse". (1962), (in corso di stampa).

Loner B. I., Optimization of manued orbilal salellite reliele design will respect 10 arlificial gravily. "As]) Tech. Rep). 61-688", (1)ee. 1961).

Iyess J., Brosio rematis on the use of planls as biological gas porehanger in "closed system. "I. Aviat. IIed. ", 25, 40ī, (1954).

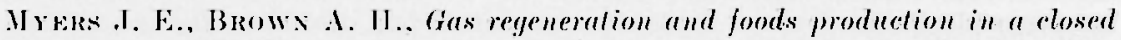
ecological system. "Nat. Acart. Sc.i., N. ('. R. n, Publ. 893, Washington, (1961).

S.A.s.A., Conference on medical resulls of the first $U$. S. manned suborbilal spece /light, Washington. D. C.. June 1961.

Pakix V. V., Some results of physsiologiral studies of man's space flighl, "C. R. Xe Congrès Eur. Méd. Aéron. Cosmont. ", l'aris, (1961).

Papes IV. O., Wasle recorery processes for a elosed ecological system. "Xat. Acad. Se., X. C. R.", Publ. s98, April 1961.

Propellaul-almosphere system sludy. "W:AD) Tech. Rej. 60-622 ", (March $1961)$.

ReDpex R. I., trine Collection and disposal deries for pressure suil. "As]) Tech. Rep. 61-329 ", (Aug. 1961).

Ross II. E., Green planls as almosphere regeneralors. "Bull. Brit. Interplial" Sioc. ", 2. 7, (1947).

Sarax C.. On lhe origin and planelary distribution of life. "Radiation Res. ", 15, $174,(1961)$.

SANDAce ('., Tolerance crileria for comtinuous inhalation erposure to torie malerial. 1. Eefjects on animals of 90-day e.rposure to phenol. COl $l_{4}$ and " mirlure of indole, slialole, $H_{2} S$ and mellugl-mereaplan. "Asl) Tech. Rep). (61-519)", (Oct. 1961).

SCano A., La depuration de lair dans les cabiues élanches. "d. R. Irr Congrès Momdial de Méd. Arom. n, Louvain, 1958.

Scaso A., L'iperossia. "Riv. Hed. Aeron. ", 21. 88 e segrg., (1958).

Scano A., I principali problemi biologici dellambienle di un veicolo spaziale, con particolare riguardo alla rigenerazione dell'aria. "20 Simposio delI'A.I.Y.S. ", Milano, 20 Aprile 1962.

SCANo A. Fea G., La depurazione dell aria in abilacoli spaziali. "Riv. Aeron, ", 34, 531, (1958).

Schärer K., Selecting a space cabio atmosphere. "Astronautics", 2. 5, (1959). 


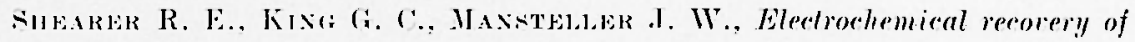
brealling orygen from earbon disoride. "Aerospace Medicine", 33, 213, (19962).

Stmoxs D. C., Manhigh II. "A F. Missile Dev. Center Tech. Rep. 59-28" (.June 1959).

Tnomas S., Hen of space, Ea. Chillon, Philadelyhia. 1960.

Tinpoxt F., Calore e temperalure al di li dell almosjera. "Riv. Aeron.", 38, 413, (1962).

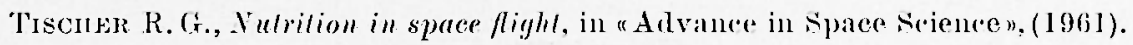

lraxny s. V., Réaction sensorielles ef dat de certaines fonctions motrices en absence de pesanteur, "(. R. Xe Congrès Eur. Méd. Aŕron. Cosmon.", Paris, (1961).

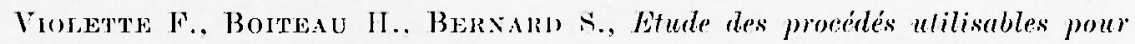
régénerer l'almosphipe au cours des voyages interplanchaires de courle durce. " IIo Congr. Mond. Med. deron. e Sp.. Roma " II. 2a, 604. (1959).

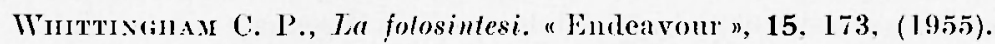

WHLARD, T. L., Researel and developmenl on closed respiralory system. accessories. Molecular sieves for carbon dionide absorplion, "ASD Tech. Rep. $61-527$ ", (Oct. 1961).

ZEEF J. D., Banbexeck R. A., Development of a unit jor recocery of water and disposal or storage of solids from human wasles. "WADD. Tech. Rep. 58-562", (Nov. 1959). 